\title{
Utility Functionals Associated With Available Congestion Control Algorithms
}

\author{
Javad Lavaei, John C. Doyle and Steven H. Low
}

\begin{abstract}
This paper is concerned with understanding the connection between the existing Internet congestion control algorithms and the optimal control theory. The available resource allocation controllers are mainly devised to derive the state of the system to a desired equilibrium point and, therefore, they are oblivious to the transient behavior of the closed-loop system. To take into account the real-time performance of the system, rather than merely its steady-state performance, the congestion control problem should be solved by maximizing a proper utility functional as opposed to a utility function. For this reason, this work aims to investigate what utility functionals the existing congestion control algorithms maximize. In particular, it is shown that there exist meaningful utility functionals whose maximization leads to the celebrated primal, dual and primal/dual algorithms. An implication of this result is that a real network problem may be solved by regarding it as an optimal control problem on which some practical constraints, such as a real-time link capacity constraint, are imposed.
\end{abstract}

\section{INTRODUCTION}

There has been a growing interest in studying the Internet congestion control ever since the first congestion collapse occurred [1]. Many algorithms have been proposed in the literature to allocate the available network resources in a fair manner among the competing users, without overloading the network. The main idea behind all these algorithms is more or less the same: each user measures some feedback signal, such as packet loss or queueing delay, and accordingly adapts its transmission rate. Among the existing transmission control protocols (TCP) for congestion control, one can name TCP-Tahoe, Reno, New Reno, and Vegas [2], [3]. More complete surveys of this topic can be found in [4], [5] and [6].

The seminal works [7] and [8] sparked remarkable process in mathematical modeling and analysis of the Internet congestion control. This advancement is due to the convex programming theory, which allows for solving a utility maximization problem by means of the Lagrangian technique. The available resource allocation algorithms, such as the primal, dual and primal/dual algorithms, are particularly designed to solve the underlying problem in a distributed way asymptotically. In other words, these algorithms guarantee that the asymptotic transmission rate of each user is the fairest rate that can be utilized without congesting the network. Having regarded the network as a system, this result implies that the control system possesses a unique globally asymptotically stable equilibrium point that corresponds to the solution of

Javad Lavaei and John C. Doyle are with the Department of Control and Dynamical Systems, California Institute of Technology (emails: lavaei@cds.caltech.edu, doyle@cds.caltech.edu).

Steven H. Low is with Computer Science and Electrical Engineering Departments, California Institute of Technology, (email: slow@caltech.edu). the static utility maximization problem. Nonetheless, it is not clear how well the system operates during its transient time. As a result, the capacity link constraints can, for instance, be violated in this period. Furthermore, these algorithms have not been derived in such a way that they can be generalized systematically to include real-time constraints such as a link capacity requirement. This work aims to revisit the congestion control problem from the standpoint of the optimal control theory.

This paper proves that the controllers proposed by the primal, dual and primal/dual algorithms all maximize some meaningful dynamical behaviors. More precisely, there exist natural utility functionals whose maximization leads to these celebrated controllers. This result opens the possibility of tackling network problems directly as optimal control problems, which not only take the dynamics into account, but which also allow to impose physical constraints. Other applications of dealing with utility functionals directly are in deducing the stability of the control system for free, gaining insight into how to perform joint routing and congestion control, etc. It is noteworthy that the development of this work relies on the inverse optimal control theory, which has a very ancient history [9], [10].

The paper is organized as follows. Some preliminaries are provided in Section II, followed by an outline of the motivations of this work in Section III. The dual algorithm is studied in Section IV using optimal control techniques, which is extended to the primal and primal/dual algorithms in Sections V and VI.

\section{PRELiminaries}

Consider a network with the set of sources $\mathcal{S}$ and the set of links $\mathcal{L}$, where each source is identified by an origin and a destination between which data can be transferred. For every $r \in \mathcal{S}$, let $x_{r}$ denote the transmission rate corresponding to source $r$ and $\mathcal{L}(r)$ denote the collection of links belonging to its fixed route. Assume that each link $l \in \mathcal{L}$ has a finite capacity $c_{l}$. Form a vector of transmission rates, denoted by $\mathbf{x}$, where its $r^{\text {th }}$ element is equal to $x_{r}$ for all $r \in \mathcal{S}$. The resource allocation problem is concerned with solving the optimization

$$
\max _{\mathbf{x}} \sum_{r \in \mathcal{S}} U_{r}\left(x_{r}\right)
$$

subject to

$$
\begin{array}{r}
\sum_{r: l \in \mathcal{L}(r)} x_{r} \leq c_{l}, \quad \forall l \in \mathcal{L} \\
x_{r} \geq 0, \quad \forall r \in \mathcal{S},
\end{array}
$$


where $U_{r}: \Re \rightarrow \Re$ is a strictly concave, increasing and twice differentiable utility function associated with source $r$. Define $R$ to be a routing matrix whose $(l, r)$ entry $(r \in$ $\mathcal{S}, l \in \mathcal{L})$ is equal to 1 if $l \in \mathcal{L}(r)$, and is 0 otherwise. Define also the aggregate flow rate $y_{l}$, the route price $q_{r}$ and the Lagrangian $L(\mathbf{x}, \mathbf{p})$ as

$$
\begin{aligned}
y_{l} & :=\sum_{r:} x_{r}, \quad l \in \mathcal{L}(r) \\
q_{r} & :=\sum_{l \in \mathcal{L}(r)} p_{l}, \quad r \in \mathcal{S}, \\
L(\mathbf{x}, \mathbf{p}) & :=\sum_{r \in \mathcal{S}} U_{r}\left(x_{r}\right)-\sum_{l \in \mathcal{L}} p_{l}\left(y_{l}-c_{l}\right),
\end{aligned}
$$

where $\mathbf{p}$ is the vector of Lagrange multipliers $p_{l}, l \in \mathcal{L}$. The Karush-Kuhn-Tucker (KKT) conditions for the utility maximization problem are

$$
\begin{aligned}
U^{\prime}\left(x_{r}\right) & =q_{r}, \\
p_{l}\left(y_{l}-c_{l}\right) & =0, \\
y_{l}-c_{l} & \leq 0, \\
x_{r}, p_{l} & \geq 0,
\end{aligned}
$$

for all $l \in \mathcal{L}$ and $r \in \mathcal{S}$. Having assumed that $R$ has full row rank, the above KKT equations have a unique solution $\left(\mathbf{x}^{*}, \mathbf{p}^{*}\right)$ [5]. Since each user $r \in \mathcal{S}$ must obtain its optimal transmission rate $x_{r}^{*}$ in terms of the available local information, a number of distributed algorithms have been proposed in the literature to enable every user to adaptively find its optimal transmission rate. One of these algorithms is briefly outlined in the sequel.

\section{A. Dual algorithm}

Assume that each link $l \in \mathcal{L}$ updates its associated price $p_{l}$ based on the rule

$$
\dot{p}_{l}(t)=h_{l}\left(p_{l}(t)\right)\left(y_{l}(t)-c_{l}\right)_{p_{l}(t)}^{+},
$$

where $h_{l}: \Re \rightarrow \Re^{+}$is a given non-decreasing continuous function and

$$
\left(y_{l}(t)-c_{l}\right)_{p_{l}(t)}^{+}=\left\{\begin{array}{cc}
y_{l}(t)-c_{l} & p_{l}(t)>0 \\
\max \left(y_{l}(t)-c_{l}, 0\right) & p_{l}(t)=0
\end{array}\right.
$$

Moreover, suppose that the user of each source $r \in \mathcal{S}$ is provided with the aggregate price along its route to update its transmission rate as

$$
x_{r}(t)=U_{r}^{\prime-1}\left(q_{r}(t)\right)
$$

It is well-known that the interconnected system specified by (5) and (7) is globally asymptotically stable with the unique equilibrium point $\left(\mathbf{x}^{*}, \mathbf{p}^{*}\right)[5]$.

\section{MOTIVATION AND PROBLEM FORMULATION}

The main idea behind the existing congestion control algorithms is to contrive a distributed control system which has a unique equilibrium point $\left(\mathbf{x}^{*}, \mathbf{p}^{*}\right)$ that is globally asymptotically stable. However, this interesting technique is oblivious to the transient behavior of the system and merely targets its steady-state behavior. As a result, the link capacity constraints may be violated during the transient time. Moreover, these indirect congestion control algorithms cannot be generalized systematically. For instance, it is pragmatic to impose a buffer size constraint or to assume that each source has a certain amount of data to transfer. These practical constraints, along with many other ones, cannot be incorporated into the aforementioned algorithms in light of the fact that these algorithms essentially rely on the static utility maximization problem to which these constraints cannot be applied. By regarding the network as a system with a specific topology, a question arises as to whether one can define an optimal control problem for this system whose solution leads to a distributed controller solving the utility maximization problem. This paper aims to show that the answer to this fundamental question is affirmative, and that working directly with the network problems in the context of optimal control theory allows the designer to incorporate other physical constraints and deduce some properties for free such as stability.

The objective is to prove that the updating policies proposed by the primal, dual and primal/dual algorithms can all be obtained by maximizing appropriate utility functionals which take the transient response of the system into account. Nevertheless, it is well-understood that even though an optimal control problem normally has a unique solution, there might be an infinite number of optimal control problems which all lead to the same solution. For instance, consider the simple first-order system $\dot{p}(t)=x(t)$, where $p(t)$ and $x(t)$ are its state and input, respectively. Note that although $x(t)$ is a standard notation for representing the state of a system, this paper needs to use this notation to denote the input of a system (as it corresponds to the transmission rate that acts as an input). Given positive numbers $k$ and $T$, there exists a unique controller that maximizes the utility functional

$$
-\int_{0}^{T}\left(\frac{x(t)^{2}}{k}+k p(t)^{2}\right) d t-p(T)^{2} .
$$

This controller turns out to be $x(t)=-k p(t)$. However, there are other utility functionals whose maximization leads to this controller. For example, the trivial term $(x(t)+k p(t))^{2}$ can be added to the integrand of the above utility functional without altering the optimal solution. It can be shown in this example that all such functionals can be characterized systematically, provided the terminal utility is fixed as $-p(T)^{2}$. To be more precise, assume that the maximization of the utility functional

$$
\int_{0}^{T} g(p(t), x(t)) d t-p(T)^{2}
$$

yields the controller $x(t)=-k p(t)$, where $g(p(t), x(t))$ is some appropriate function. One can verify that there exist a function $\hat{g}(p(t), x(t))$ and a constant number $\mu$ such that

$$
g(p(t), x(t))=\mu+\hat{g}(p(t), x(t))-\frac{x(t)^{2}}{k}-k p(t)^{2},
$$

where $\hat{g}(p(t), x(t))$ is equal to zero along all trajectories of the optimal closed-loop system. This simple toy example 
implies that there are an infinite number of utility functionals which solve the inverse optimal problem; nevertheless, they all share some key part that determines the trade-off between the state and the input which has caused the optimal controller to be identical to the given one.

The above discussion signifies that there might be numerous utility functionals associated with the static utility maximization problem. The primary objective is to identify their common part that has meaningful physical interpretations. It will be later shown that there is a close parallel (term by term) between the functionals solving the utility maximization problem and the ones characterized in (9) and (10).

\section{OPTIMAL CONTROL FOR DUAL ALGORITHM}

Having provided each user $r$ with its route price that is obtained based on some pre-specified rule, assume that the user is required to find the best updating policy to adjust its transmission rate $x_{r}$. This hypothesis implies that the dynamical system

$$
\dot{p}_{l}(t)=h_{l}\left(p_{l}(t)\right)\left(y_{l}(t)-c_{l}\right)_{p_{l}(t)}^{+}, \quad l \in \mathcal{L}
$$

exists in the core of the network to generate link prices, where $\mathbf{p}(t)$ and $\mathbf{x}(t)$ are the state and the input of the system, respectively. It is desired to find a utility functional whose maximization leads to the local controllers

$$
x_{r}(t)=U_{r}^{\prime-1}\left(q_{r}(t)\right), \quad r \in \mathcal{S} .
$$

\section{A. Simple illustrative example}

Before handling the problem in the general case, let the main ideas be elucidated in a very simple example. As a trivial but illustrative case, assume that:

- The network has only one source and one link.

- The capacity of the link is equal to 1 .

- The utility function $U(x)$ is equal to $-0.5(x-4)^{2}$ if $x \in[0,3]$.

- The weighting function $h(p)$ is identical to 1 .

Note that since $\mathcal{S}$ and $\mathcal{L}$ each have one element, the indices $l$ and $r$ are omitted. Moreover, although the utility function $U(x)$ is defined only on the interval of interest $[0,3]$, it can be extended smoothly to the entire interval $[0, \infty)$. For simplicity, suppose that the value of the initial price $p(0)$ is chosen so that the transmission rate $x(t)$ always stays in the interval $[0,3]$, and that the price $p(t)$ never hits zero. The problem now reduces to finding a utility functional whose maximization leads to the controller

$$
x(t)=-q(t)+4
$$

for the system

$$
\dot{q}(t)=x(t)-1 .
$$

In order to eliminate the constant terms in the above equations, introduce the change of variables

$$
\begin{aligned}
& \bar{x}(t)=x(t)-1, \\
& \bar{q}(t)=q(t)-3 .
\end{aligned}
$$

In the new coordinates, the system and the controller turn out to be $\dot{\bar{q}}(t)=\bar{x}(t)$ and $\bar{x}(t)=-\bar{q}(t)$, respectively. This control system has been studied in the toy example of the previous section (assuming $k=1$ ), for which the utility functional

$$
-\int_{0}^{T}\left(\bar{x}(t)^{2}+\bar{q}(t)^{2}\right) d t-\bar{q}(T)^{2}
$$

was found. One can rewrite the above expression in terms of the original variables to obtain

$$
-\int_{0}^{T}\left((x(t)-1)^{2}+(q(t)-3)^{2}\right) d t-(q(T)-3)^{2} .
$$

To relate the terms in the above functional to the static utility maximization problem, notice that

$$
\begin{aligned}
3-q(t) & =U^{\prime-1}(q(t))-1=\arg \max _{v} L(v, q(t)) \\
(q(T)-3)^{2} & =2 \max _{v} L(v, q(T))+9 .
\end{aligned}
$$

Substituting the above relations into (17), one can conclude that maximizing the utility functional given below leads to the dual controller:

$$
\begin{aligned}
& -\frac{1}{2} \int_{0}^{T}\left((x(t)-c)^{2}+\left(\arg \max _{v} L(v, q(t))-c\right)^{2}\right) d t \\
& -\max _{v} L(v, q(T)) .
\end{aligned}
$$

As can be inferred from the toy example in Section III, every other utility functional that is able to solve the underling inverse optimal problem includes the integrand of the above functional, in addition to some trivial terms, provided its terminal utility is chosen as above. This result will be generalized in the sequel, and the interpretation of the individual terms appearing in this utility functional will then be discussed in detail.

\section{B. General case}

The next theorem extends the above-mentioned results to the general case.

Theorem 1: Given $T>0$, the decentralized controller given in (12) maximizes the utility functional

$$
\begin{aligned}
\max _{\mathbf{x}(t)}\{ & \frac{1}{2} \int_{0}^{T} \sum_{l \in \mathcal{L}}\left\{Y_{l}\left(y_{l}(t), p_{l}(t)\right)+Y_{l}\left(\tilde{y}_{l}(\mathbf{p}(t)), p_{l}(t)\right)\right\} d t \\
& \left.-\max _{\mathbf{v}(T)} L(\mathbf{v}(T), \mathbf{p}(T))\right\}
\end{aligned}
$$

for the system (11), where

$$
Y_{l}\left(\alpha, p_{l}(t)\right):=-\left(\alpha-c_{l}\right) h_{l}\left(p_{l}(t)\right)\left(\alpha-c_{l}\right)_{p_{l}(t)}^{+}
$$

for every $\alpha \in \Re, l \in \mathcal{L}$, and

$$
\tilde{\mathbf{y}}(\mathbf{p}(t)):=R \times \arg \max _{\mathbf{v}(t)} L(\mathbf{v}(t), \mathbf{p}(t))
$$

$\left(\tilde{y}_{l}(\mathbf{p}(t))\right.$ is equal to the $l^{\text {th }}$ entry of $\left.\tilde{\mathbf{y}}(\mathbf{p}(t))\right)$. 
Proof: Define the optimal cost-to-go function $J(\mathbf{p}, t), t \in$ $[0, T]$, to be

$$
\begin{aligned}
& J(\mathbf{p}, t):=\max _{\mathbf{x}(s)}\left\{\frac { 1 } { 2 } \int _ { t } ^ { T } \sum _ { l \in \mathcal { L } } \left\{Y_{l}\left(y_{l}(s), p_{l}(s)\right)\right.\right. \\
& \left.\left.+Y_{l}\left(\tilde{y}_{l}(\mathbf{p}(s)), p_{l}(s)\right)\right\} d s-\max _{\mathbf{v}(T)} L(\mathbf{v}(T), \mathbf{p}(T))\right\},
\end{aligned}
$$

where the system starts at time $t$ with an initial state $\mathbf{p}$ whose entries are all nonnegative. The Hamilton-Jacobi-Bellman (HJB) method [11] states that $J(\mathbf{p}, t)$ satisfies the partial differential equation

$$
\begin{aligned}
0 & =\frac{\partial J(\mathbf{p}, t)}{\partial t}+\max _{\mathbf{x}}\left\{\frac{1}{2} \sum_{l \in \mathcal{L}}\left\{Y_{l}\left(y_{l}, p_{l}\right)+Y_{l}\left(\tilde{y}_{l}(\mathbf{p}), p_{l}\right)\right\}\right. \\
& \left.+\sum_{l \in \mathcal{L}} h_{l}\left(p_{l}\right)\left(y_{l}-c_{l}\right)_{p_{l}}^{+} \frac{\partial J(\mathbf{p}, t)}{\partial p_{l}}\right\}
\end{aligned}
$$

with the boundary condition

$$
J(\mathbf{p}, T)=-\max _{\mathbf{v}} L(\mathbf{v}, \mathbf{p}) .
$$

Solving the HJB differential equation is cumbersome in general. However, it is desired to show that this equation takes the simple solution $J(\mathbf{p}, t)=J(\mathbf{p}, T), \forall t \in[0, T]$ in this problem. To this end, observe that

$$
\tilde{y}_{l}(\mathbf{p})=\sum_{r: l \in \mathcal{L}(r)} U_{r}^{\prime-1}\left(q_{r}\right) .
$$

Since $\mathbf{p}$ is a nonnegative vector, the maximum of the Lagrangian $L(\mathbf{v}, \mathbf{p})$ (with respect to $\mathbf{v}$ ) is achieved when

$$
v_{r}=U_{r}^{\prime-1}\left(q_{r}\right), \quad r \in \mathcal{S},
$$

where $v_{r}$ denotes the $r^{\text {th }}$ entry of $\mathbf{v}$, for all $r \in \mathcal{S}$. For the above-mentioned choice of $J(\mathbf{p}, t)$, it can be verified that

$$
\begin{aligned}
& \frac{\partial J(\mathbf{p}, t)}{\partial t}=0, \\
& \frac{\partial J(\mathbf{p}, t)}{\partial p_{l}}=\tilde{y}_{l}(\mathbf{p})-c_{l}, \quad \forall l \in \mathcal{L} .
\end{aligned}
$$

Using these equalities, one can also check that the input $\mathbf{x}$ given by

$$
x_{r}=U_{r}^{\prime-1}\left(q_{r}\right), \quad r \in \mathcal{S}
$$

maximizes the objective functional

$$
\begin{aligned}
& \frac{1}{2} \sum_{l \in \mathcal{L}}\left\{Y_{l}\left(y_{l}, p_{l}\right)+Y_{l}\left(\tilde{y}_{l}(\mathbf{p}), p_{l}\right)\right\} \\
& +\sum_{l \in \mathcal{L}} h_{l}\left(p_{l}\right)\left(y_{l}-c_{l}\right)_{p_{l}}^{+} \frac{\partial J(\mathbf{p}, t)}{\partial p_{l}}
\end{aligned}
$$

with respect to $\mathrm{x}$. By substituting the equations (28) and (29) into (24), it is straightforward to observe that the equation (24) is satisfied. Hence, the HJB method implies that the controller given in (29) (after replacing $\left(x_{r}, q_{r}\right)$ with $\left.\left(x_{r}(t), q_{r}(t)\right)\right)$ is an optimal controller for the underlying system.

The utility functional given in Theorem 1 has several interesting features that will be spelled out next. Consider the price vector $\mathbf{p}(t)$ at a time instant $t \in[0, T]$. The best transmission rates that the users may utilize at this time can be obtained by maximizing the term $L(\mathbf{v}(t), \mathbf{p}(t))$ over all possible $\mathbf{v}(t)$ 's. In other words, $\arg \max _{\mathbf{v}(t)} L(\mathbf{v}(t), \mathbf{p}(t))$ is indeed the optimal instantaneous transmission rates that the system can accept given its current link prices. As a result, the terminal utility $\max _{\mathbf{v}(T)} L(\mathbf{v}(T), \mathbf{p}(T))$ resembles the static Lagrangian at time $T$, but is maximized over all possible transmission rates to evaluate the potential of the system given its final price $\mathbf{p}(T)$. In other words, a variant of the static utility maximization problem is mainly integrated into the final utility (and partially incorporated into the integrand to take care of the transient behavior). On the other hand, the integrand has two terms $Y_{l}\left(y_{l}(t), p_{l}(t)\right)$ and $Y_{l}\left(\tilde{y}_{l}\left(\mathbf{p}(t), p_{l}(t)\right)\right.$, each of which has a physical interpretation. The term $Y_{l}\left(y_{l}(t), p_{l}(t)\right)$ can be regarded as the actual $l^{\text {th }}$ link utility at time $t$, by virtue of the following observations:

- If $p_{l}(t)$ is nonzero, then $Y_{l}\left(y_{l}(t), p_{l}(t)\right)$ is proportional to the quadratic term $-\left(y_{l}(t)-c_{l}\right)^{2}$, which implies that in order not to over-utilize or under-utilize the network, the best strategy is to maintain the flow rate $y_{l}(t)$ precisely at the capacity of the link.

- If $p_{l}(t)$ is zero, then $Y_{l}\left(y_{l}(t), p_{l}(t)\right)$ indicates that the optimal utilization of the link corresponds to employing a flow rate below the link capacity.

Furthermore, $Y_{l}\left(\tilde{y}_{l}\left(\mathbf{p}(t), p_{l}(t)\right)\right.$ can be envisaged as the virtual $l^{\text {th }}$ link utility at time $t$ due to the fact that $\tilde{y}_{l}(\mathbf{p}(t))$ is the optimal transmission rate over the $l^{\text {th }}$ link given the current price $\mathbf{p}(t)$. To summarize the ideas, the proposed utility functional is natural in the sense it maximizes the sum of the actual and virtual link utilities over the time interval $[0, T)$ and a variant of the static utility function at the final time $T$.

Corollary 1: For every time instant $T>0$, the following relation holds:

$$
\begin{aligned}
& \max _{\mathbf{x}(t)}\{ \frac{1}{2} \int_{0}^{T} \sum_{l \in \mathcal{L}}\left\{Y_{l}\left(y_{l}(t), p_{l}(t)\right)+Y_{l}\left(\tilde{y}_{l}(\mathbf{p}(t)), p_{l}(t)\right)\right\} d t \\
&\left.-\max _{\mathbf{v}(T)} L(\mathbf{v}(T), \mathbf{p}(T))\right\}=-\max _{\mathbf{v}(0)} L(\mathbf{v}(0), \mathbf{p}(0)) .
\end{aligned}
$$

Proof: It follows from the proof of Theorem 1 and the HJB equation that the expression given in the left side of the equality (31) is identical to the optimal cost-to-go $J(\mathbf{p}(0), 0)$. On the other hand, it is shown in the proof of Theorem 1 that $J(\mathbf{p}(0), 0)$ is equal to the right side of the above equation. This completes the proof.

Theorem 1 and corollary 1 assert that there exists a natural utility functional whose maximization leads to the celebrated dual TCP controller, and that the maximum value of this functional is equal to $-\max _{\mathbf{v}(0)} L(\mathbf{v}(0), \mathbf{p}(0))$. As pointed out earlier, this term corresponds to the maximum source utility at time $t=0$ under the given initial price $\mathbf{p}(0)$.

Evidently, there are some utility functionals that trivially solve the inverse optimal problem under study. For instance, 
one candidate is

$$
-\int_{0}^{\infty} \sum_{r \in \mathcal{S}}\left(x_{r}(t)-U_{r}^{\prime-1}\left(q_{r}(t)\right)\right)^{2} d t
$$

Nevertheless, this utility functional has nothing to do with the static utility maximization problem, and provides no extra information about the system such as its closed-loop stability. In contrast, Theorem 1 proposes a meaningful utility functional, which is somewhat involved. A question arises as to whether there exists a simpler utility functional which still conveys meaningful interpretations. To answer this question, notice that the terminal utility given in (20) is a suitable counterpart of the original static utility function. Therefore, it remains to show that the integrand of this functional is essentially required and cannot be simplified. For this purpose, assume that the controller (12) maximizes the utility functional

$$
\max _{\mathbf{x}(t)}\left\{\int_{0}^{T} g(\mathbf{p}(t), \mathbf{x}(t)) d t-\max _{\mathbf{v}(T)} L(\mathbf{v}(T), \mathbf{p}(T))\right\}
$$

for the system (11), where $T$ is a positive time and $g(\mathbf{p}(t), \mathbf{x}(t))$ is some function. Suppose also that $g(\mathbf{p}, \mathbf{x})$ is continuously differentiable at every point $(\mathbf{p}, \mathbf{x})$ for which $\mathbf{p}$ is strictly positive. Define the optimal cost-to-go function $J(\mathbf{p}, t)$ as

$$
J(\mathbf{p}, t):=\int_{t}^{T} g(\tilde{\mathbf{p}}(s), \tilde{\mathbf{x}}(s)) d s-\max _{\mathbf{v}(T)} L(\mathbf{v}(T), \tilde{\mathbf{p}}(T)),
$$

where $\tilde{\mathbf{p}}(s)$ and $\tilde{\mathbf{x}}(s)$ denote the state and the input of the system (11) under the controller (12) in the case when the system starts at time $t$ with the initial state p. Finally, assume that $J(\mathbf{p}, t)$ is continuously differentiable with respect to $\mathbf{p}$ and $t$.

Theorem 2: Under the assumptions made above, there exist a function $\hat{g}(\mathbf{p}(t), \mathbf{x}(t))$ and a real number $\mu$ such that

$$
\begin{aligned}
& g(\mathbf{p}(t), \mathbf{x}(t))=\mu+\hat{g}(\mathbf{p}(t), \mathbf{x}(t)) \\
& +\frac{1}{2} \sum_{l \in \mathcal{L}}\left\{Y_{l}\left(y_{l}(t), p_{l}(t)\right)+Y_{l}\left(\tilde{y}_{l}(\mathbf{p}(t)), p_{l}(t)\right)\right\},
\end{aligned}
$$

where the function $\hat{g}(\mathbf{p}(t), \mathbf{x}(t))$ is identically zero along all trajectories of the optimal closed-loop system.

Proof: In light of the assumptions made right before Theorem 2, one can write the HJB equation for this system as

$$
\begin{aligned}
0 & =\frac{\partial J(\mathbf{p}, t)}{\partial t}+\max _{\mathbf{x}}\{g(\mathbf{p}, \mathbf{x}) \\
& \left.+\sum_{l \in \mathcal{L}} h_{l}\left(p_{l}\right)\left(y_{l}-c_{l}\right)_{p_{l}}^{+} \frac{\partial J(\mathbf{p}, t)}{\partial p_{l}}\right\},
\end{aligned}
$$

where $J(\mathbf{p}, t)$ is given in (34). Consider a strictly positive vector $\mathbf{p}$. Taking the derivative of the above expression with respect to $x_{r}, r \in \mathcal{S}$, yields

$$
\sum_{l \in \mathcal{L}(r)} h_{l}\left(p_{l}\right) \frac{\partial J(\mathbf{p}, t)}{\partial p_{l}}=-\frac{\partial g(\mathbf{p}, \mathbf{x})}{\partial x_{r}} .
$$

Since $R$ has full row rank, the quantities $\frac{\partial J(\mathbf{p}, t)}{\partial p_{l}}, l \in \mathcal{L}$, can be uniquely solved in terms of $\frac{\partial g(\mathbf{p}, \mathbf{x})}{\partial x_{r}}, r \in \mathcal{S}$. This result, together with the memoryless property of the controller (12), implies that $\frac{\partial J(\mathbf{p}, t)}{\partial p_{l}}$ does not depend on time. Hence, it follows from the HJB equation that $\frac{\partial J(\mathbf{p}, t)}{\partial t}$ does not depend on time either. As a result, there exist a scalar $\mu$ and a function $f(\mathbf{p})$ such that

$$
J(\mathbf{p}, t)=f(\mathbf{p})-\mu t .
$$

On the other hand, the boundary condition on the HJB equation states that

$$
J(\mathbf{p}, T)=-\max _{\mathbf{v}} L(\mathbf{v}, \mathbf{p}) .
$$

Thus, one can conclude that

$$
J(\mathbf{p}, t)=-\max _{\mathbf{v}} L(\mathbf{v}, \mathbf{p})-\mu(t-T), \quad \forall \mathbf{p}>0 .
$$

It follows from the continuity of $J(\mathbf{p}, t)$ that

$$
J(\mathbf{p}, t)=-\max _{\mathbf{v}} L(\mathbf{v}, \mathbf{p})-\mu(t-T), \quad \forall \mathbf{p} \geq 0 .
$$

Having written $g(\mathbf{p}, \mathbf{x})$ in the form of (35), substituting the above equation into the HJB equation yields that the function $\hat{g}(\mathbf{p}(t), \mathbf{x}(t))$ is equal to zero along all trajectories of the optimal closed-loop system. This completes the proof.

Notice that the term $\hat{g}(\mathbf{p}(t), \mathbf{x}(t))$ in Theorem 2 is a trivial term, which provides no useful information. This quantity can be, for instance, equal to the integrand of the trivial utility functional (32). Ignoring the uninformative terms $\mu$ and $\hat{g}(\mathbf{p}(t), \mathbf{x}(t))$, the functional given in Theorem 2 reduces to the one provided in Theorem 1 .

It can be observed that the utility functionals characterized in Theorem 2 closely parallel those provided in (9) and (10) for a simple toy example. More specifically:

- $Y_{l}\left(y_{l}(t), p_{l}(t)\right)$ corresponds to $-\frac{x(t)^{2}}{k}$. This term depends much more weakly on the state, but strongly on the input.

- $Y_{l}\left(\tilde{y}_{l}(t), p_{l}(t)\right)$ corresponds to $-k p(t)^{2}$, which only penalizes the state.

- The constant term $\mu$ exists in both utility functionals.

- $\hat{g}(\mathbf{p}(t), \mathbf{x}(t))$ corresponds to $\hat{g}(p(t), x(t))$, which is an uninformative term and specifies no trade-off between the state and the input.

\section{Stability proof}

An application of the optimal control problem introduced in Theorem 1 is that the global asymptotic stability of the system (11) under the static controller (12) can be concluded automatically.

Theorem 3: The controller (12) that maximizes the utility functional (20) for the system (11) makes the pair $(\mathbf{x}(t), \mathbf{p}(t))$ converge to the fixed point $\left(\mathbf{x}^{*}, \mathbf{p}^{*}\right)$.

Proof: The main idea behind the proof is to observe that

$$
\begin{aligned}
Y_{l}\left(y_{l}(t), p_{l}(t)\right) & \leq 0, \quad \forall t \in[0, T], l \in \mathcal{L}, \\
Y_{l}\left(\tilde{y}_{l}(\mathbf{p}(t)), p_{l}(t)\right) & \leq 0, \quad \forall t \in[0, T], l \in \mathcal{L}, \\
-\max _{\mathbf{v}(T)} L(\mathbf{v}(T), \mathbf{p}(T)) & \leq-\mathbf{p}^{*},
\end{aligned}
$$


and that the state and input of the closed-loop control system satisfy the equation (by Corollary 1)

$$
\begin{aligned}
& \frac{1}{2} \int_{0}^{T} \sum_{l \in \mathcal{L}}\left\{Y_{l}\left(y_{l}(t), p_{l}(t)\right)+Y_{l}\left(\tilde{y}_{l}(\mathbf{p}(t)), p_{l}(t)\right)\right\} d t \\
& -\max _{\mathbf{v}(T)} L(\mathbf{v}(T), \mathbf{p}(T))=-\max _{\mathbf{v}(0)} L(\mathbf{v}(0), \mathbf{p}(0)) .
\end{aligned}
$$

By letting $T$ go to infinity, the relations (42) and (43) can be combined to conclude that

$$
\begin{aligned}
Y_{l}\left(y_{l}(t), p_{l}(t)\right) \rightarrow 0 \quad \text { as } \quad t \rightarrow \infty, \\
Y_{l}\left(\tilde{y}_{l}(\mathbf{p}(t)), p_{l}(t)\right) \rightarrow 0 \quad \text { as } \quad t \rightarrow \infty,
\end{aligned}
$$

for every $l \in \mathcal{L}$, in light of the fact that the left side of the equation (43) must remain finite and cannot go to $-\infty$ due to the finiteness of its right side. As a result

$$
\lim _{t \rightarrow \infty}\left(y_{l}(t)-c_{l}\right)_{p_{l}(t)}^{+}=0, \quad \forall l \in \mathcal{L} .
$$

The proof follows immediately from the above equation.

\section{Joint routing and congestion control}

It is desired to accomplish both routing and resource allocation simultaneously. For this purpose, assume that each source has a fixed origin and destination, but an undetermined route. The objective is to find an optimal route for every source so that the utility of the network is maximized. Note that since the Lagrangian introduced in (3) depends on the unknown routing matrix $R$, it will be denoted by $L(\mathbf{x}, \mathbf{p} ; R)$ henceforth. As far as the optimal routing with respect to the static utility function is concerned, one should solve the optimization problem

$$
\max _{R} \min _{\mathbf{p}} \max _{\mathbf{x}} L(\mathbf{x}, \mathbf{p} ; R)=\max _{R} L\left(\mathbf{x}^{*}(R), \mathbf{p}^{*}(R) ; R\right)
$$

to find $R$, where $\left(\mathrm{x}^{*}(R), \mathbf{p}^{*}(R)\right)$ is the saddle point of the Lagrangian in the case when the routing matrix of the network is $R$. Solving the above optimization problem in a distributed way is formidable, because it is NP-hard even at the centralized level [12]. Aside from this point, a static utility function may not be a good measure for optimal routing, as the transient behavior of the system should also be taken into account. In what follows, the problem of optimal routing with respect to the utility functional (20) is addressed.

Theorem 4: Given $r \in \mathcal{S}$, find all possible simple paths in the network which starts from the origin of source $r$ and ends at the destination of this source. For each of these paths, compute the initial route price, i.e. the route price based on the link price vector $\mathbf{p}(0)$. Among these paths, each one with the minimum initial price is an optimal route for source $r$ under the problem of joint routing and congestion control with respect to the utility functional (20).

Proof: The joint routing and resource allocation with respect to the utility functional (20) amounts to solving the optimization problem

$$
\begin{aligned}
& \max _{R} \min _{\mathbf{x}(t)}\left\{\frac { 1 } { 2 } \int _ { 0 } ^ { T } \sum _ { l \in \mathcal { L } } \left\{-Y_{l}\left(y_{l}(t), p_{l}(t)\right)\right.\right. \\
& \left.\left.-Y_{l}\left(\tilde{y}_{l}(\mathbf{p}(t)), p_{l}(t)\right)\right\} d t+\max _{\mathbf{v}(T)} L(\mathbf{v}(T), \mathbf{p}(T) ; R)\right\},
\end{aligned}
$$

which is tantamount to (by Corollary 1 )

$$
\max _{R} \max _{\mathbf{v}(0)} L(\mathbf{v}(0), \mathbf{p}(0) ; R) \text {. }
$$

Denote the optimal routing matrix with $R^{*}$. Besides, define $q_{r}(t ; R), r \in \mathcal{S}$, as the route price associated with source $r$ at time $t$ under the routing matrix $R$. It is evident that

$$
\begin{aligned}
\max _{\mathbf{v}(0)} & L(\mathbf{v}(0), \mathbf{p}(0) ; R)=\sum_{r \in \mathcal{S}}\left\{U_{r}\left(U_{r}^{\prime-1}\left(q_{r}(0 ; R)\right)\right)\right. \\
& \left.-q_{r}(0 ; R) U_{r}^{\prime-1}\left(q_{r}(0 ; R)\right)\right\}+\sum_{l \in \mathcal{L}} c_{l} p_{l}(0) .
\end{aligned}
$$

Note that the terms $q_{r_{1}}(0 ; R)$ and $q_{r_{2}}(0 ; R)$ are independent of each other for every $r_{1}, r_{2} \in \mathcal{S}$ such that $r_{1} \neq r_{2}$, due to the fact that they are contingent upon different columns of $R$. Hence, in order to maximize the expression given in the above relation over all possible routing matrices $R$, the following optimization problem can be solved alternatively:

$$
\max _{R}\left\{U_{r}\left(U_{r}^{\prime-1}\left(q_{r}(0 ; R)\right)\right)-q_{r}(0 ; R) U_{r}^{\prime-1}\left(q_{r}(0 ; R)\right)\right\}
$$

For a scalar variable $q$, one can write

$$
\begin{aligned}
& \frac{\partial\left(U_{r}\left(U_{r}^{\prime-1}(q)\right)-q U_{r}^{\prime-1}(q)\right)}{\partial q}=U_{r}^{\prime}\left(U_{r}^{\prime-1}(q)\right) \frac{\partial U_{r}^{\prime-1}(q)}{\partial q} \\
& -U_{r}^{\prime-1}(q)-q \frac{\partial U_{r}^{\prime-1}(q)}{\partial q}=-U_{r}^{\prime-1}(q) \leq 0 .
\end{aligned}
$$

This means that the function

$$
U_{r}\left(U_{r}^{\prime-1}(q)\right)-q U_{r}^{\prime-1}(q)
$$

is non-increasing in the variable $q$. As a result, it can be concluded from (50) that $q_{r}\left(0 ; R^{*}\right)$ is equal to the minimum of $q_{r}(0 ; R)$ over all possible routing matrices $R$. This completes the proof.

Consider a network over which both routing and congestion control are to be performed. If the users of network were fixed and remained online for a very long time, it could be justified that an optimal route should be obtained based on the optimal equilibrium point of the system. However, since users in a real network join and leave, and most of the sources do not live long, it is reasonable to take the transient behavior of the system into account for optimal routing. Under this circumstance, Theorem 4 proves that the optimal routing is really simple and intuitive: each user who joins the network should find a route to its destination whose initial price is minimum. Note that it is commonly accepted in the literature that routing could be performed by assigning a cost to each link and then minimizing the route cost. The present work 
shows that this simple idea indeed leads to an optimal route taking care of the transient response of the system.

\section{E. Another meaningful utility functional}

Roughly speaking, the utility functional proposed in Theorem 1 treats a variant of the static utility function as the terminal utility and defines dynamical utility functions on the links. Another idea would be to define dynamical utility functions on the sources. This idea has been exploited in the next theorem.

Theorem 5: Assume that the weighting functions $h_{l}\left(p_{l}(t)\right), l \in \mathcal{L}$ are all equal to 1 . Given $T>0$, the decentralized controller (12) maximizes the utility functional

$$
\begin{aligned}
\max _{\mathbf{x}(t)}\{ & \int_{0}^{T}\left(\sum_{r \in \mathcal{S}} U_{r}\left(x_{r}(t)\right)-\max _{\mathbf{v}(t)} L(\mathbf{v}(t), \mathbf{p}(t))\right) d t \\
& \left.-\frac{1}{2} \mathbf{p}(T)^{T} \mathbf{p}(T)\right\}
\end{aligned}
$$

for the system (11). Furthermore, the maximum of this utility functional is equal to $-\frac{1}{2} \mathbf{p}(0)^{T} \mathbf{p}(0)$.

Proof: The proof can be carried out in line with that of Theorem 1 after noticing that the optimal cost-to-go function for this control problem is equal to $J(\mathbf{p}, t)=-\frac{1}{2} \mathbf{p}^{T} \mathbf{p}$. The details are omitted here for brevity.

The utility functional proposed in Theorem 5 has an interesting interpretation. The quantity $\max _{\mathbf{v}(t)} L(\mathbf{v}(t), \mathbf{p}(t))$ is equal to the maximum instantaneous source utility that the system can provide based on the price $\mathbf{p}(t)$. Hence, the integrand $\sum_{r \in \mathcal{S}} U_{r}\left(x_{r}(t)\right)-\max _{\mathbf{v}(t)} L(\mathbf{v}(t), \mathbf{p}(t))$ can be regarded as the relative source utility function. Having assumed $h_{l}\left(p_{l}(t)\right)$ to be equal to 1 , each price $p_{l}(t)$ can be visualized as the queue size at the buffer of the $l^{\text {th }}$ router. Thus, the utility functional provided in the theorem aims to maximize the relative utility function over the time interval $[0, T)$ and minimize the sum of routers' queue sizes at the final time $T$. The maximum of the utility functional, which is equal to the negative half of the sum of the squared queue sizes at $t=0$, is independent of the route. This property, together with the negative term inside the integrand, does not allow for deducing the stability of the dual control system from this functional for free, or searching for the optimal route.

\section{OPTIMAL CONTROL FOR PRIMAL ALGORITHM}

Let the utility maximization problem stated in Section II be modified as

$$
\max _{\mathbf{x}}\left\{\sum_{r \in \mathcal{S}} U_{r}\left(x_{r}\right)-\sum_{l \in \mathcal{L}} \int_{0}^{y_{l}} f_{l}(y) d y\right\},
$$

where $f_{l}(y)$ is a barrier function that can be interpreted as the price for transferring data at the rate $y$ on link $l$. Assume that $f_{l}(\cdot), l \in \mathcal{L}$, is a non-decreasing, continuous function such that

$$
\int_{0}^{y_{l}} f_{l}(y) d y \rightarrow \infty \quad \text { as } \quad y_{l} \rightarrow \infty
$$

Furthermore, assume that $U_{r}\left(x_{r}\right), r \in \mathcal{S}$, goes to $-\infty$ as $x_{r}$ approaches zero. Under these assumptions, the above utility maximization problem has a unique solution $\mathrm{x}^{*}$ at which the gradient of $V(\mathbf{x})$ vanishes, where

$$
V(\mathbf{x})=\sum_{r \in \mathcal{S}} U_{r}\left(x_{r}\right)-\sum_{l \in \mathcal{L}} \int_{0}^{y_{l}} f_{l}(y) d y .
$$

To obtain the solution $\mathrm{x}^{*}$ in a distributed way, consider the interconnected system given by

$$
\dot{x}_{r}(t)=k_{r}\left(x_{r}(t)\right)\left(U_{r}^{\prime}\left(x_{r}(t)\right)-q_{r}(t)\right), \quad \forall r \in \mathcal{S}
$$

and

$$
p_{l}(t)=f_{l}\left(y_{l}(t)\right), \quad \forall l \in \mathcal{L},
$$

where $k_{r}: \Re \rightarrow \Re^{+}$is a non-decreasing continuous function. It is known that the point $\left(\mathbf{x}^{*}, \mathbf{p}^{*}\right)$ is the globally asymptotically stable fixed point of this interconnected system [5]. Thus, the above distributed system can be run to asymptotically solve the static utility maximization problem. The objective is to find the optimal control counterpart of this result. For this purpose, assume that the memoryless system (58) exists in the core of the network to generate the link prices, and that each user deploys a simple integrator to adjust its transmission rate as

$$
\dot{x}_{r}(t)=u_{r}(t), \quad r \in \mathcal{S},
$$

where $u_{r}(t)$ is some input signal that needs to be determined. It is noteworthy that $p_{l}(t)$ is a measured output of this system. The goal is to derive a utility functional for the system (59) whose maximization leads to the decentralized controller

$$
u_{r}(t)=k_{r}\left(x_{r}(t)\right)\left(U_{r}^{\prime}\left(x_{r}(t)\right)-q_{r}(t)\right), \quad r \in \mathcal{S} .
$$

Theorem 6: Given a time instant $T>0$, the decentralized controller (60) maximizes the utility functional

$$
\begin{aligned}
\max _{\mathbf{u}(t)} & \left\{-\frac{1}{2} \int_{0}^{T}\left(\mathbf{u}(t)^{T} \mathbf{K}(\mathbf{x}(t))^{-1} \mathbf{u}(t)\right.\right. \\
+ & \left.\left.\nabla V(\mathbf{x}(t))^{T} \mathbf{K}(\mathbf{x}(t)) \nabla V(\mathbf{x}(t))\right) d t+V(\mathbf{x}(T))\right\}
\end{aligned}
$$

for the system given by (58) and (59), where

- $\mathbf{K}(\mathbf{x}(t))$ is a diagonal matrix with the $(r, r)$ diagonal entry $k_{r}\left(x_{r}(t)\right)$ for all $r \in \mathcal{S}$.

- $\mathbf{u}(t)$ is a vector with the $r^{\text {th }}$ entry $u_{r}(t)$ for all $r \in \mathcal{S}$.

- The symbol $\nabla$ denotes the gradient operator.

Moreover, the maximum of this utility functional is equal to $V(\mathbf{x}(0))$.

Proof: One can adopt the technique used in Theorem 1 to prove this theorem, after considering the optimal cost-to-go function $J(\mathbf{x}, t)$ as $V(\mathbf{x})$.

As before, the utility functional proposed in the above theorem has some plausible intrinsic properties. For instance, this functional treats the static utility function as a terminal utility, and encompasses two terms accounting for the transient behavior of the system. The term 
$\nabla V(\mathbf{x}(t))^{T} \mathbf{K}(\mathbf{x}(t)) \nabla V(\mathbf{x}(t))$ penalizes the nonzero gradient of the objective function $V(\mathbf{x}(t))$ during the transient time (note that the optimal solution of the static utility maximization problem corresponds to the unique point at which the gradient of $V(\mathbf{x})$ vanishes). Besides, the term $\mathbf{u}(t)^{T} \mathbf{K}(\mathbf{x}(t))^{-1} \mathbf{u}(t)$ or equivalently $\dot{\mathbf{x}}(t)^{T} \mathbf{K}(\mathbf{x}(t))^{-1} \dot{\mathbf{x}}(t)$ is a measure of users' willingness to alter their transmission rates abruptly. Thus, $\mathbf{K}(\mathbf{x})$ is a weighting function representing the trade-off between the above penalty terms.

In analogy with Theorem 3, the stability of the system (59) under the control (60) is an immediate consequence of Theorem 6. More precisely, since the integrand of the proposed utility functional is always less than or equal to zero and its terminal utility is bounded from above by $V\left(\mathbf{x}^{*}\right)$, letting $T$ grow towards infinity yields

$$
\nabla V(\mathbf{x}(t))^{T} \mathbf{K}(\mathbf{x}(t)) \nabla V(\mathbf{x}(t)) \rightarrow 0 \quad \text { as } \quad t \rightarrow \infty
$$

or equivalently

$$
\|\nabla V(\mathbf{x}(t))\| \rightarrow 0 \quad \text { as } t \rightarrow \infty .
$$

It results from the above relation that the state of the closed-loop system converges to the unique maximizer of the function $V(\mathbf{x})$.

\section{A. Joint routing and congestion control}

It is desired to perform joint routing and congestion control for the primal controller similar to what was carried out in Section IV-D for the dual controller. To this end, since the utility function $V(\mathbf{x})$ depends on the routing matrix, it will be denoted by $V(\mathbf{x} ; R)$ henceforth. An optimal routing matrix with respect to the utility function (56) can be obtained by solving the optimization problem

$$
\max _{R} \max _{\mathbf{x}} V(\mathbf{x} ; R),
$$

which may be a cumbersome distributed optimization problem. In contrast, an optimal routing matrix with respect to the utility functional given in Theorem 6 can be found by solving

$$
\max _{R} V(\mathbf{x}(0) ; R)
$$

or equivalently

$$
\min _{R} \sum_{l \in \mathcal{L}} \int_{0}^{R_{l} \mathbf{x}(0)} f_{l}(y) d y
$$

where $R_{l}, l \in \mathcal{L}$, denotes the $l^{\text {th }}$ row of $R$. It is evident that the above optimization problem is far simpler than the one given in (64). For instance, if $f_{l}(y)$ is equal to $y$ for every $l \in \mathcal{L}$, then an optimal routing matrix $R$ can be obtained by solving the optimization problem $\min _{R}\|R \mathbf{x}(0)\|_{2}$, where $\|\cdot\|_{2}$ denotes the 2-norm operator. Recall that the joint routing and congestion control for the dual controller causes each source to take a minimum-price route, which may not be a proper strategy as several sources could take the same route and some possible routes may remain empty. In contrast, the joint routing and congestion control for the primal controller makes every effort that each link is not over-utilized at the initial time.

\section{B. Congestion control and multi-path routing}

Assume that there could exist multiple routes between each source-destination pair. Denote the source of route $r \in \mathcal{S}$ with $s(r)$. The utility maximization problem in this case can be regarded as the maximization of the utility function

$$
V(\mathbf{x})=\sum_{i \in \mathcal{S}} U_{i}\left(\sum_{r: s(r)=i} x_{r}\right)-\sum_{l \in \mathcal{L}} \int_{0}^{y_{l}} f_{l}(y) d y .
$$

It is known that the maximization of the above function could give rise to more than one solution [5]. In the case when there exists a unique maximizer, the optimal transmission rates can be obtained asymptotically using the distributed controller

$$
\dot{x}_{r}(t)=u_{r}(t), \quad \forall r \in \mathcal{S},
$$

where

$$
u_{r}(t)=k_{r}\left(U_{s(r)}^{\prime}\left(\sum_{p: s(p)=s(r)} x_{p}(t)\right)-q_{r}(t)\right),
$$

and $k_{r}$ is an arbitrary positive number. The question arises as to what meaningful utility functional the above primal controller maximizes. To answer this question, it can be shown that one such a functional is the utility functional given in Theorem 6 for single-path routing, but with $V(\mathbf{x})$ provided in (67) as opposed to the one in (56). This shows that the multi-path routing case is a simple extension of the single-path routing case. Now, different properties, such as stability, can be deduced as before.

\section{OPTIMAL CONTROL FOR PRIMAL/DUAL ALGORITHM}

Consider an interconnected system consisting of the subsystem (11) in the core of the network to generate prices and the subsystem (59) at the edge of the network to adjust the transmission rates. The states of this system are $\mathbf{x}(t)$ and $\mathbf{p}(t)$, while its input (to be found) is $\mathbf{u}(t)$. The goal of this part is to obtain a utility functional whose maximization yields the distributed controller (60). The techniques developed earlier can be exploited to tackle this problem. It can be shown that one such optimal control problem can be defined as

$$
\begin{aligned}
\max _{\mathbf{u}(t)}\{ & -\frac{1}{2} \int_{0}^{T}\left(\nabla_{x} L(\mathbf{x}(t), \mathbf{p}(t))^{T} \mathbf{K}(\mathbf{x}(t)) \nabla_{x} L(\mathbf{x}(t), \mathbf{p}(t))\right. \\
& \left.+\mathbf{u}(t)^{T} \mathbf{K}(\mathbf{x}(t))^{-1} \mathbf{u}(t)-2 \sum_{l \in \mathcal{L}} Y_{l}\left(y_{l}(t), p_{l}(t)\right)\right) d t \\
& +L(\mathbf{x}(T), \mathbf{p}(T))\},
\end{aligned}
$$

where $\nabla_{x}$ denotes the gradient operator with respect to the first argument $\mathbf{x}$. The integrand of this functional is the difference between those given for the primal and dual algorithms (if $\tilde{y}_{l}(\mathbf{p}(t))$ is identified by $y_{l}(t)$ ). However, physical intuition suggests that a good utility functional for this case should be the sum of those obtained for the dual and primal algorithms separately (as opposed to their difference). Indeed, the term $\sum_{l} Y_{l}\left(y_{l}(t), p_{l}(t)\right)$ in the above 
utility functional is a measure of link utility (as pointed out earlier) that is minimized, instead of being maximized. This phenomenon can be justified by noticing that the static utility maximization problem is a min-max optimization (as performed on the Lagrangian), whereas the above utility functional is only a max optimization. It is worth noting that the utility functional proposed in Theorem 1 is also a min-max optimization. Obtaining a better utility functional for this case is left for future research.

\section{CONCLUSIONS}

This work relates the optimal control theory to the Internet congestion control algorithms. The main motivation for investigating this relationship is that the existing algorithms solve the utility maximization problem only at the equilibrium point and ignore the transient behavior of the control system. Therefore, they cannot be modified systematically to incorporate other physical constraints, such as a real-time link capacity requirement. In order to substantiate that the optimal control theory provides the right tools to solve a constrained network utility problem in practice, it is shown that there exist natural, meaningful utility functionals whose maximization yields the distributed controllers proposed by the primal, dual and primal/dual algorithms. These utility functionals provide useful insights into the optimal closedloop system; for instance, they automatically conclude the closed-loop stability for free.

\section{ACKNOWLEDGMENT}

This research was supported by ONR MURI N00014-081-0747 "Scalable, Data-driven, and Provably-correct Analysis of Networks," ARO MURI W911NF-08-1-0233 “Tools for the Analysis and Design of Complex Multi-Scale Networks," and the Army's W911NF-09-D-0001 Institute for Collaborative Biotechnology.

\section{REFERENCES}

[1] V. Jacobson and M. J. Karels, "Congestion avoidance and control," ACM Computer Communication Review, vol. 18, no. 4, pp. 341-329, 1988.

[2] L. L. Peterson and B. S. Davie, "Computer networks: a systems approach," Morgan Kaufman, 1999.

[3] L. S. Bramko and L. L. Peterson, "TCP Vegas: end-to-end congestion avoidance on a global Internet," IEEE Journal on Selected Areas in Communications, vol. 13, no. 8, pp. 1465-1480, 1995.

[4] S. Shakkottai and R. Srikant, "Network optimization and control," Foundations and Trends in Networking, vol. 2, no. 3, pp. 271-379, 2008.

[5] R. Srikant, "The mathematics of Internet congestion control," Birkhauser, 2004.

[6] M. Chiang, S. H. Low, A. R. Calderbank and J. C. Doyle, "Layering as optimization decomposition," in Proceedings of IEEE, vol. 95, no. 1, pp. 255-312, 2007.

[7] F. P. Kelly, "Charging and rate control for elastic traffic," European Transactions on Telecommunications, vol. 8, no. 1, pp. 33-37, 1997.

[8] F. P. Kelly, A. Maullo and D. Tan, "Rate control in communication networks: shadow prices, proportional fairness and stability," Journal of the Operational Research Society, vol. 49, pp. 237-252, 1998.

[9] W. Rugh, "On an inverse optimal control problem," IEEE Transactions on Automatic Control, vol. 16, no. 1, pp. 87-88, 1971.

[10] P. Moylan and B. Anderson, "Nonlinear regulator theory and an inverse optimal control problem," IEEE Transactions on Automatic Control, vol. 18 , no. 5, pp. 460-465, 1973.
[11] D. E. Kirk, "Optimal control theory: an introduction," Dover Publications, 2004.

[12] J. Wang, L. Li, S. H. Low and J. C. Doyle, "Cross-layer optimization in TCP/IP networks," IEEE/ACM Transactions on Networking, vol. 13 , no. 3, pp. 582-268, 2005. 